Received: 18 June 2018

Accepted: 4 September 2018

Published online: 27 September 2018

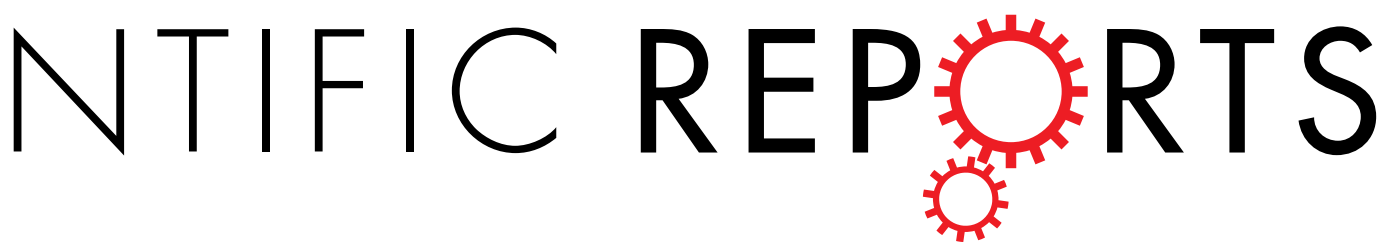

\title{
OPEN A requirement for slc15a4 in imiquimod-induced systemic inflammation and psoriasiform inflammation in mice
}

\author{
Alexis D. Griffith ${ }^{1}$, Asifa K. Zaidi ${ }^{1}$, Ashley Pietro ${ }^{1}$, Matthew Hadiono ${ }^{1}$, Jessica S. Yang ${ }^{1}$, \\ Rachel Davis ${ }^{1}$ \& Daniel L. Popkin ${ }^{1,2}$
}

There is competing evidence that plasmacytoid dendritic cells (pDC), the most potent source of IFN-I, may initiate psoriasis. We targeted pDC function using the slc15a 4 feeble loss-of-function mouse whose PDC are unresponsive to TLR agonists. slc15a $4^{\text {feeble }}$ treated with the topical TLR7-agonist imiquimod (IMO) demonstrated decreased epidermal thickening 24 hours post-treatment which was more pronounced by day 5 as compared to wildtype mice. These findings were specific to the acute IMO model and not the protracted IL23 model that drives inflammation downstream of TLR activation. Systemically, slc15a4 was required for IMQ-induced weight loss and cutaneous accumulation of CD4+ and Siglec $\mathrm{H}+$, but not CD11b + cells. Consistent with this phenotype and the function of slc15a4, induction of IFN-I was virtually absent systemically and via cutaneous gene expression. Induction of other inflammatory cytokines (cytokine storm) was modestly blunted in slc15a $4^{\text {feeble }}$ except for inflammasome-associated genes consistent with slc15a4 being required for TLR7-mediated (but not inflammasome-mediated) inflammation downstream of IMO. Surprisingly, only IFN-I gene expression was suppressed within IMQ-treated skin. Other genes including conserved psoriasiform trademark gene expression were augmented in slc15a feeble versus littermate controls. Taken together, we have identified a role for slc15a4 but not canonical psoriasiform genes in the imiquimod model of psoriasiform dermatitis.

Psoriasis is a chronic autoimmune disease affecting approximately $2 \%$ of the population ${ }^{1}$. While the cause of psoriasis is unknown, there is competing evidence that plasmacytoid dendritic cells (pDC) may be central to disease development $\mathrm{t}^{2-5}$. pDCs are responsible for producing $\sim 1000$ times more type I interferon (IFN-I) than any other cell type. Thus, despite comprising only $\sim 1 \%$ of the already rare dendritic cell population, pDCs account for approximately $50 \%$ of all circulating IFN-I following infection ${ }^{6}$. It has been proposed that pDCs in genetically predisposed individuals under certain environmental conditions and through their secretion of IFN-I, activate autoimmune $\mathrm{T}$ cells, in turn, eliciting a psoriasiform phenotype $\mathrm{e}^{4}$.

SIc15a4. Solute carrier gene family 15 member 4 (SLC15A4) is a twelve membrane spanning protein also known as peptide/histidine transporter 1 (PHT1). Slc15a4 cellular gene expression essentially mirrors TLR7 and 9 expression being most highly expressed in antigen presenting cells, specifically plasmacytoid dendritic cells (pDC) and B cells ${ }^{7-9}$. Consistently, a functional role for slc15a4 has only been demonstrated in these two cell types to date. Slc15a4 contains an acidic dileucine motif which likely mediates localization to the endosomal compartment via AP- $3^{7}$. Subsequent biogenesis of lysosome-related organelles (LROs) harboring TLR7 and TLR9 is defective without slc15a4. Thus TLR7 and TLR9 sensing is specifically lost in slc15a4 mutant mice resulting in acute loss of IFN-I production secondary to defective $\mathrm{pDC}$ within 6 hours post CpG injection ${ }^{7}$ and loss of B cell functional responses reported as early as 48 hours after TLR7 and TLR9 stimulation ${ }^{10}$.

${ }^{1}$ Department of Dermatology, Case Western Reserve University Hospitals, Cleveland, OH, 44106, USA. ${ }^{2}$ Departments of Dermatology, Pathology, Molecular Biology and Microbiology, Case Western Reserve University Hospitals, Cleveland, $\mathrm{OH}, 44106$, USA. Correspondence and requests for materials should be addressed to D.L.P. (email: daniel. popkin@case.edu) 
slc15a4 phenotypes in vivo: SLE, acute and chronic viral infection. Consistent with the specific function and expression of slc15a4, slc15a $4^{\text {feeble }}$ loss of function mice are protected in the NZB and Fas(lpr) mouse models of systemic lupus erythematosus (SLE) ${ }^{11}$. GWAS studies have likewise identified slc15a4 as a genetic locus contributing to human lupus in 6 independent studies ${ }^{12-17}$.

The physiological role of $s l c 15 a 4$ is likely for protection against pathogens via TLR7/9 sensing. We tested this hypothesis and found that slc15a4 was not required for control of acute viremia but was necessary for virus-specific $\mathrm{T}$ cell activation and chronic viral clearance ${ }^{18}$. Here we extend our analysis of slc15a4 to examine its role in psoriasiform dermatitis mediated by the canonical TLR7 agonist imiquimod as compared to intradermal IL23 injection.

Imiquimod (IMQ) model, TLR7, IFN-I and plasmacytoid DC (pDC). The IMQ model of acute skin inflammation has become the most widely used mouse model in this category over the past several years ${ }^{19}$. TLR7 sensing via $\mathrm{pDC}$ is thought to drive massive IFN-I expression within 6 hours that contributes to the induction of multiple inflammatory markers and histologic alterations in the skin seen within $12-24$ hours ${ }^{19}$. However, there is also a vehicle-specific TLR7-independent effect which activates the inflammasome and contributes in part to the acanthosis and inflammation present in $\operatorname{skin}^{20,21}$. pDC production of IFN-I is an expected dramatic component of the IMQ model and critical to initiate psoriasis in a xenograft model ${ }^{4}$. However, deficiency of the IFN-I receptor was reported to have no effect in two IMQ studies ${ }^{5,20}$, while two subsequent IMQ studies demonstrated

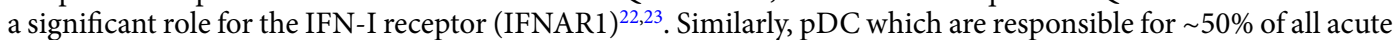
IFN- $\mathrm{I}^{24}$ has been implicated in psoriasis ${ }^{4}$ and the IMQ psoriasiform model. Yet surprisingly, targeting these cells did not result in any significant changes in the IMQ model ${ }^{5}$.

Here, we evaluated the role of slc15a4 in imiquimod-induced psoriasiform dermatitis. The mouse mutation examined in our study (feeble) has a one base-pair mutation in slc15a4 rendering the transporter inactive but feeble mice are otherwise genetically identical to their C57BL/6 J wildtype littermates (WT). Consistently, these mice are indistinguishable from WT during development, including DC and B cell phenotypic populations. However, there is a dramatic loss of DC function in response to TLR stimulation, especially regarding IFN-I secretion ${ }^{7,10}$ as well as a defect in IgG2c class switch recombination during CpG-adjuvanted vaccination ${ }^{10}$. Using this feeble model, we found significant decreases in disease initiation and progression in slc15a4feeble as compared to WT mice both locally and systemically.

\section{Results}

The role of pDC and IFN-I in the IMQ psoriasiform dermatitis model is controversial in addition to the source of IFN-I production ${ }^{4,5,20,22,23}$. Therefore, we examined an alternative target which may also be inhibited therapeutically to treat TLR-pDC driven immune pathology, slc15a4. Slc15a4 is required for pDC TLR7/9 sensing and subsequent TLR driven IFN-I production ${ }^{7}$. We and others found that slc15a4 was important in disease mod$\mathrm{els}^{8,11,18,25}$. Therefore, we hypothesized that studying slc15a4 deficiency in the IMQ psoriasiform dermatitis model may clarify the role of the TLR7-pDC-IFN-I pathway of immune pathology. A significant role would also justify slc15a4 as a specific therapeutic target to alleviate disease.

slc15a4 feeble mice are more resistant to imiquimod induced weight loss than epidermal skin thickening in an acute psoriasiform dermatitis model, but not resistant to IL23 induced skin inflammation. We applied IMQ or vehicle control to the shaved backs of WT and slc15a4feeble (slc15a4 deficient mice) for 4 consecutive days and terminated the experiment on day 5 to be consistent with most IMQ studies $^{19}$. Dramatically, slc15a feeble mice grossly appeared unaffected by IMQ, i.e. no hunching, withered appearance or decrease in movement was observed in contrast to their WT littermates. Consistently, IMQ-induced weight loss was not observed in slc15a4feeble as compared to WT mice (Fig. 1A). Similarly, skin punch biopsies demonstrated attenuated acanthosis in slc15a4feeble compared to WT mice as early as 24 hours post IMQ (Fig. 1B). This difference in skin thickening was greater by day 5 (Fig. 1B and C). To determine the specificity of slc15a4 in this psoriasiform model, we executed a more chronic model of psoriasiform dermatitis that activates the downstream inflammatory cascade by direct intralesional injection of IL23 which promotes Th17 skewing. We found that after 11 days, slc15a4feeble had increased ear skin thickness as compared to their WT littermates (Fig. 2A) and acanthosis similar to that induced by IMQ in WT mice (Fig. 2B). Thus, we reasoned that a slc15a4 pro-inflammatory role was necessary in the IMQ model potentially by driving a very proximal event in the inflammatory pathway (i.e. pDC sensing of IMQ).

slc15a4 is required for IMQ-induced recruitment of CD4+ and SiglecH + cells. The IMQ model of psoriasiform dermatitis is driven by cytokine secreting CD4+ T cells of the lymphoid compartment in addition to CD11b + myeloid cells $s^{26,27}$. These cell types are critical for the local and systemic effects of IMQ that we observed (Fig. 1). Thus, we compared the inflammatory infiltrate between treated and control WT and slc15a feeble mice on day 5 of IMQ treatment to determine if there was a difference in inflammatory cell infiltration by immunohistochemistry (IHC). We also quantitated the presence of the $\mathrm{pDC}$ marker SiglecH given the specific loss of function of pDC in slc15a4 deficient mice. We observed an absence of CD4+ and SiglecH+ cells accumulating in the treated skin of slc15a4feeble mice as compared to littermate controls by day 5 (Fig. 3A,B). These data implicated $\mathrm{CD} 4+\mathrm{T}$ lymphocytes and pDC as potential cell types driving IMQ-driven weight loss and acanthosis via slc15a4. In contrast, there was an essentially normal accumulation of CD11b + cells in slc15a feeble vs. WT mice. Albeit, slc15a feeble had a slightly lower absolute number of resident CD11b+ myeloid cells at baseline (Fig. 3A).

slc15a4 is required for IFN-I induction. pDC secrete $\sim 1000 x$ more IFN-I than any other cell type. Consistently, pDC are responsible for $\sim 50 \%$ of all circulating IFN-I during acute viral infections despite being extremely rare cells ${ }^{6}$. Given the absence of SiglecH + pDC accumulating in slc15a feeble , and the central role of 

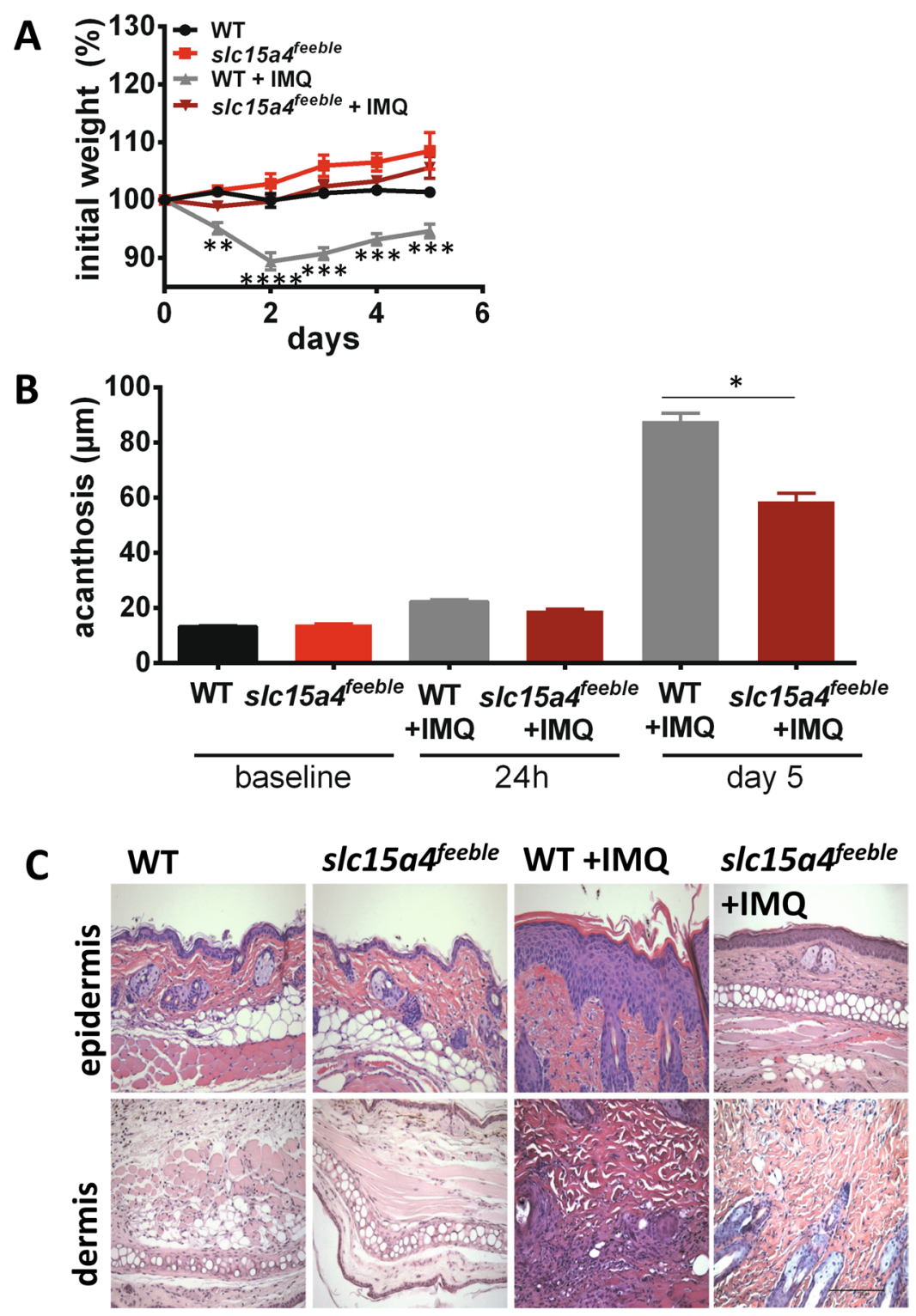

Figure 1. slc15a4 is required for imiquimod induced weight loss and epidermal thickening. slc15a4feeble and WT mice were treated with imiquimod (IMQ) and euthanized after 24 hours or 5 days. (A) Significant IMQ-induced weight loss was observed in WT mice as compared to slc15a4feeble mice $(\mathrm{n}=7,5,17,15)$. (B) Attenuated IMQ-induced acanthosis was observed in slc15a4feeble mice at 24 hours and 5 days post daily IMQ treatment compared to WT mice $(n=7,5,6,6,17,5)$. (C) Representative images of H\&E stained mouse dorsal skin from WT and slc15a4feeble mice demonstrated attenuated acanthosis in slc15a4feeble mice compared to WT mice on day $5(n=7,5,17,15)$. Images taken at $200 \times$. Scale is the same in all pictures; scale bar $=100 \mu \mathrm{m}$. Data combined from 3 independent experiments. $* \mathrm{p}<0.05, * * \mathrm{p}<0.005, * * * \mathrm{p}<0.0005, * * * * \mathrm{p}<0.0001$ in unpaired two-sided parametric T tests.

pDC in IMQ responses ${ }^{28-30}$, we hypothesized that slc15a4 would be required for IMQ-induced IFN-I. We found that the loss of slc15a4 accounted for $>1.5 \log$ decrease in IFN-I production (Fig. 4).

slc15a4 contributes to systemic cytokine storm during topical IMQ treatment. IFN-I treatment is known to cause weight loss and general malaise that is associated with general inflammation ${ }^{31}$. Thus, we reasoned that IMQ-induced systemic IFN-I would likely propagate a broad induction of circulating inflammatory cytokines ("cytokine storm"). We measured 25 cytokines to address this possibility (Fig. 5). We found a potent induction of almost all 25 cytokines by IMQ. This induction was either unchanged or attenuated in slc15a4feeble. This attenuation was not generally due to a higher steady state concentration of cytokines in slc15a4feeble as absolute plasma concentrations were overall very similar as compared to WT mice (Supp. Fig. 5). Only CXCL6(24 h and day 5), IL-1a ( $24 \mathrm{~h}$ only) and IL-6 (24h only) were increased in slc15a4 feeble as compared to WT mice. These cytokines share a common nidus, inflammasome activation ${ }^{32,33}$. Induction of IL1b (the canonical output of the inflammasome) did not meet statistical significance, although it did trend in the same direction (Fig. 5). 

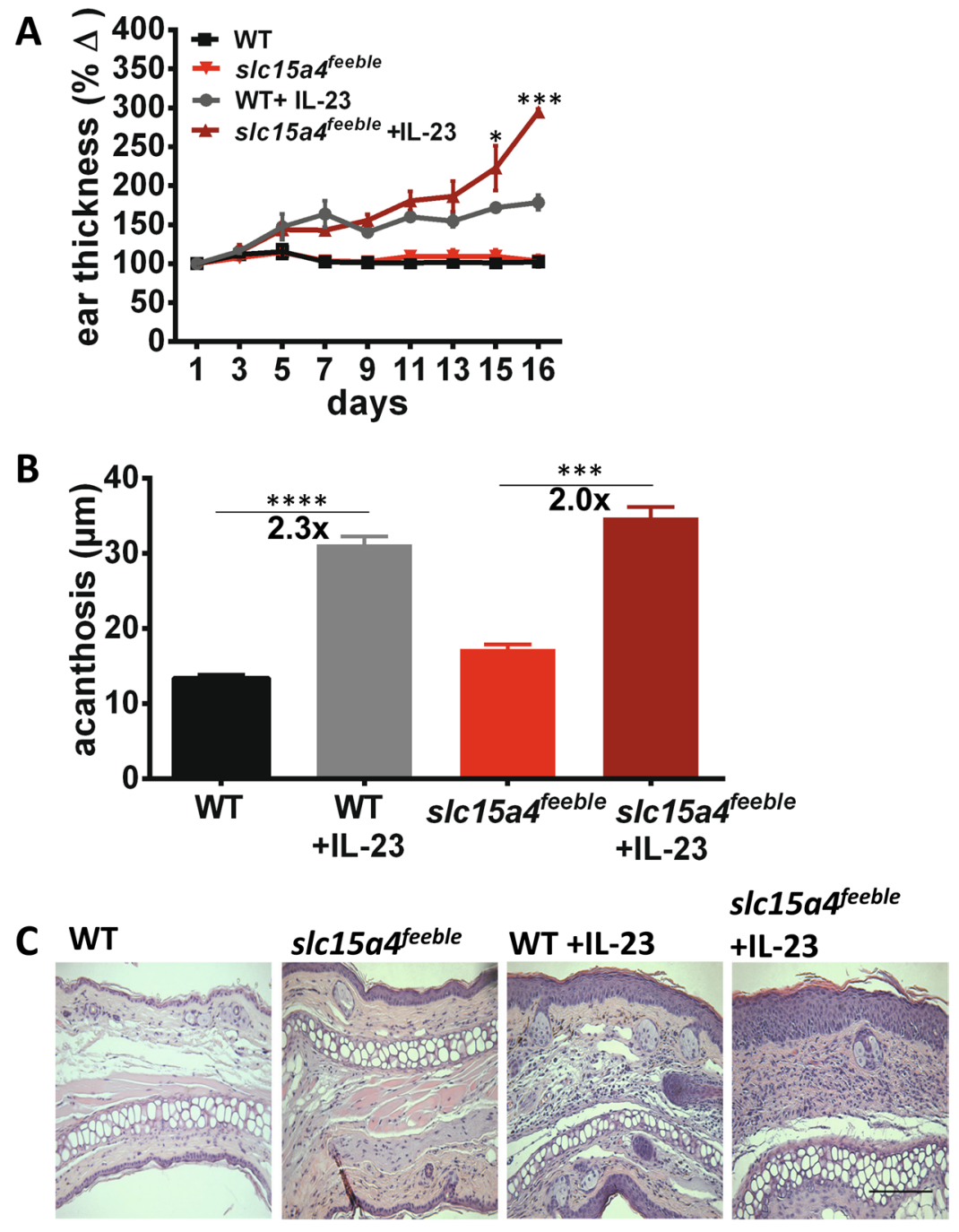

Figure 2. s1c15a $4^{\text {feeble }}$ mice exhibit increased inflammation of the ear following intralesional injections of IL23. Ears of WT and slc15a feeble mice were injected every other day for 16 consecutive days with 20uL PBS containing $500 \mathrm{ng}$ IL23. (A) On day 16, ears were collected for staining and measurement via H\&E. (B) Ears were measured every other day with a micrometer, before injections $(n=7,7,6)$. (C) Representative $H \& E$ sections of mouse ears following treatment with IL23 $(n=7,7,6,6)$. Images taken at 200x. Scale is the same in all pictures; scale bar $=100 \mu \mathrm{m}$. Data from two independent experiments each containing 6-7 mice/group. $* \mathrm{p}<0.05, * * * \mathrm{p}<0.0005, * * * * \mathrm{p}<0.0001$ in unpaired two-sided parametric T tests.

Unexpected more potent induction of trademark psoriasiform genes and inflammatory cytokines in skin of slc15a4 deficient mice. Lastly, we asked whether the IMQ-treated skin may contribute to the systemic increase in circulating cytokines. To address this we isolated total RNA from the treated skin 24 hours and on day 5 post initiation of treatment and quantitated gene expression for those cytokines identified in the luminex assay above, with validated TaqMan primer-probesets available. We added IL12b and IL23a given their central role in psoriasiform inflammation as the canonical gene products driving Th1 and Th17 skewing, respectively ${ }^{34}$. To further evaluate inflammasome associated genes, review of the literature demonstrated IL1f6 (IL-36a) and CXCL5 as additional IMQ-induced gene products ${ }^{35,36}$. RT-qPCR demonstrated induction of many of these cytokines at $24 \mathrm{~h}$ in WT mice (Fig. 6A) similar to what we observed in the plasma (Fig. 5A). However, this local skin induction was largely absent by day 5 , consistent with downstream elaboration of circulating cytokines by immune cells which have left the treated skin in WT mice (Fig. 6A). This correlation with plasma proteins included the relative increase of inflammasome-related genes in in slc15a $4^{\text {feeble }}$ compared to WT mice (Figs 5A and 6A) in addition to a moderate increase in the Th1 and Th17 skewing gene products IL12b and IL23a, respectively. Surprisingly, we found more potent gene induction of inflammatory cytokines within the skin of overall less-inflamed slc15a $4^{\text {feeble }}$ mice by day 5 (Figs 5B and 6D). This could not be generally explained by lower basal gene expression in slc15a $4^{\text {feeble }}$ mice (Fig. 6S). Taken together, we concluded that local skin gene induction of inflammatory cytokines may play a role in the first day of IMQ treatment. However, by day 5 the absence of weight loss and diminished cytokine storm in slc15a $4^{\text {feeble }}$ could not be correlated with local gene expression. Thus, we reasoned that local gene expression could not serve as the predominant source for 

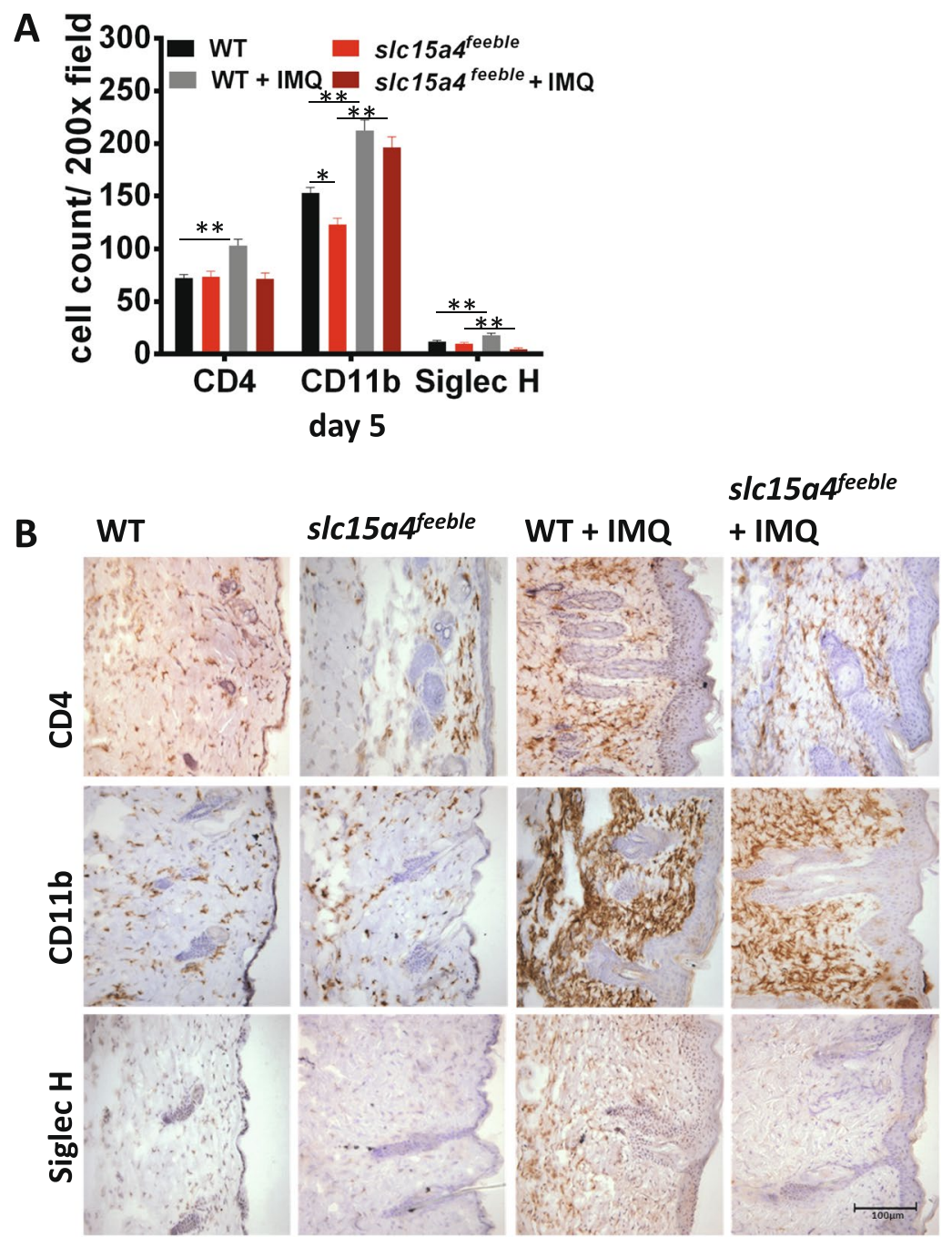

Figure 3. IMQ-dependent recruitment of CD4 $+\mathrm{T}$ cells into the dermis requires slc15a4. slc15a4 feeble and WT mice were shaved and treated with imiquimod (IMQ) or only shaved and euthanized after 5 days. (A) Quantitative measurements of CD4 T-cell (CD4), myeloid cell (CD11b) and pDC (Siglec H) infiltrate from immunostained dorsal mouse skin of WT and slc15a4feeble mice 5 days post topical treatment with IMQ or control. $\mathrm{n}=4,5,4,5$ mice for WT, slc15a4 feeble, WT + IMQ, slc15a4feeble + IMQ, respectively. (B) Representative immunostained sections of dorsal mouse skin at 6 days post treatment with IMQ. $n=4,5,4,5$ images from left to right. Images taken at 200x. Scale is the same in all pictures; scale bar $=100 \mu \mathrm{m}$. Data is from 1 of 3 similar experiments with 4-5 mice/group. ${ }^{*} \mathrm{p}<0.05,{ }^{*} \mathrm{p}<0.005$, in unpaired two-sided parametric $\mathrm{T}$ tests.

circulating cytokines on day 5 . We than measured the most strongly increased trademark psoriasisform genes conserved across humans and 5 mouse models of psoriasiform dermatitis (including IMQ-induced) ${ }^{35}$ (Fig. 6B,E).

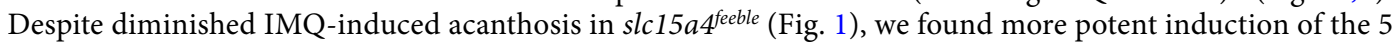
most induced genes conserved across animal models and human psoriasis at $24 \mathrm{~h}$ and this increase was maintained on day 5 (Fig. 6B,E). We then confirmed that the genes whose regulation were most diminished in magnitude and over time in $s l c 15 a 4^{\text {feeble }}$ were the type I interferon genes: Ifna 2 and Ifnb1 but not type II interferon (gamma) (Fig. 6C,F). Thus, we concluded that increased induction of psoriasis-associated genes is not sufficient for maximal skin thickening and that diminished IFN-I, both local and systemic, was the most likely culprit.

\section{Discussion}

The role of IFN-I in the IMQ model of psoriasiform dermatitis is controversial ${ }^{5,20,22,23}$. The most potent producer of IFN-I is the very rare pDC cell type. Thus efforts to target this cell type systemically for elimination can be difficult to access throughout the animal given the potential critical effects of even a few remaining cells. Additionally, compensatory responses are possible in the absence of this cell type. Therefore, we chose an alternative approach to access a potential therapeutic intervention for pDC-TLR7 driven immune pathology, genetic disruption of slc15a4. Slc15a4 is required for pDC TLR7/9 sensing and subsequent TLR driven IFN-I production ${ }^{7}$. We and others previously found that this pathway was important in disease models ${ }^{11,18,37}$. Therefore, we hypothesized that studying slc15a4 deficiency in the IMQ-induced psoriasiform dermatitis model may serve as an alternative 


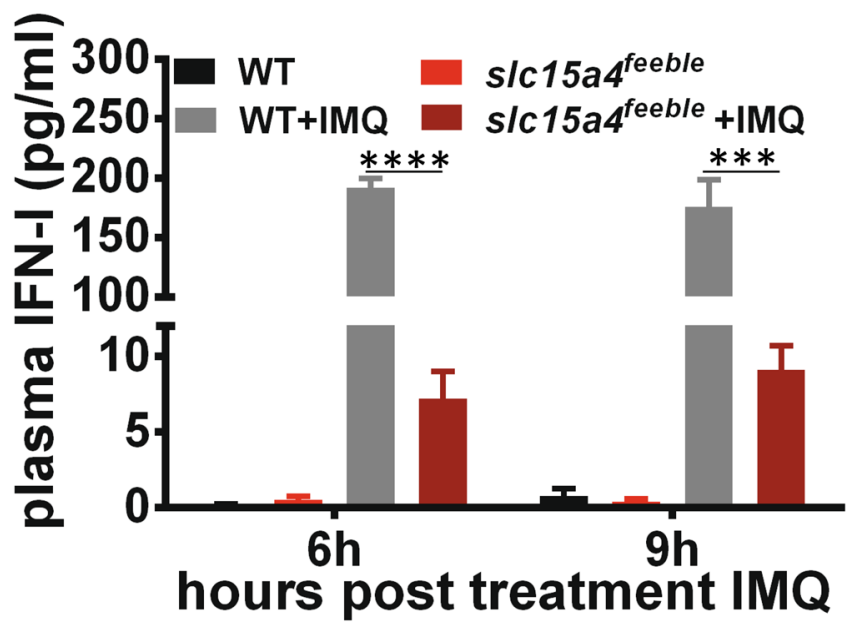

Figure 4. slc15a4 is required for type 1 interferon induction. Plasma was collected 6 and 9 hours post IMQ from WT and slc15a feeble mice. IFN-I levels were than measured by ELISA. $\mathrm{n}=4$ /group. 1 of 2 similar experiments shown. $* * * \mathrm{p}<0.0005, * * * * \mathrm{p}<0.0001$ in unpaired two-sided parametric T tests.
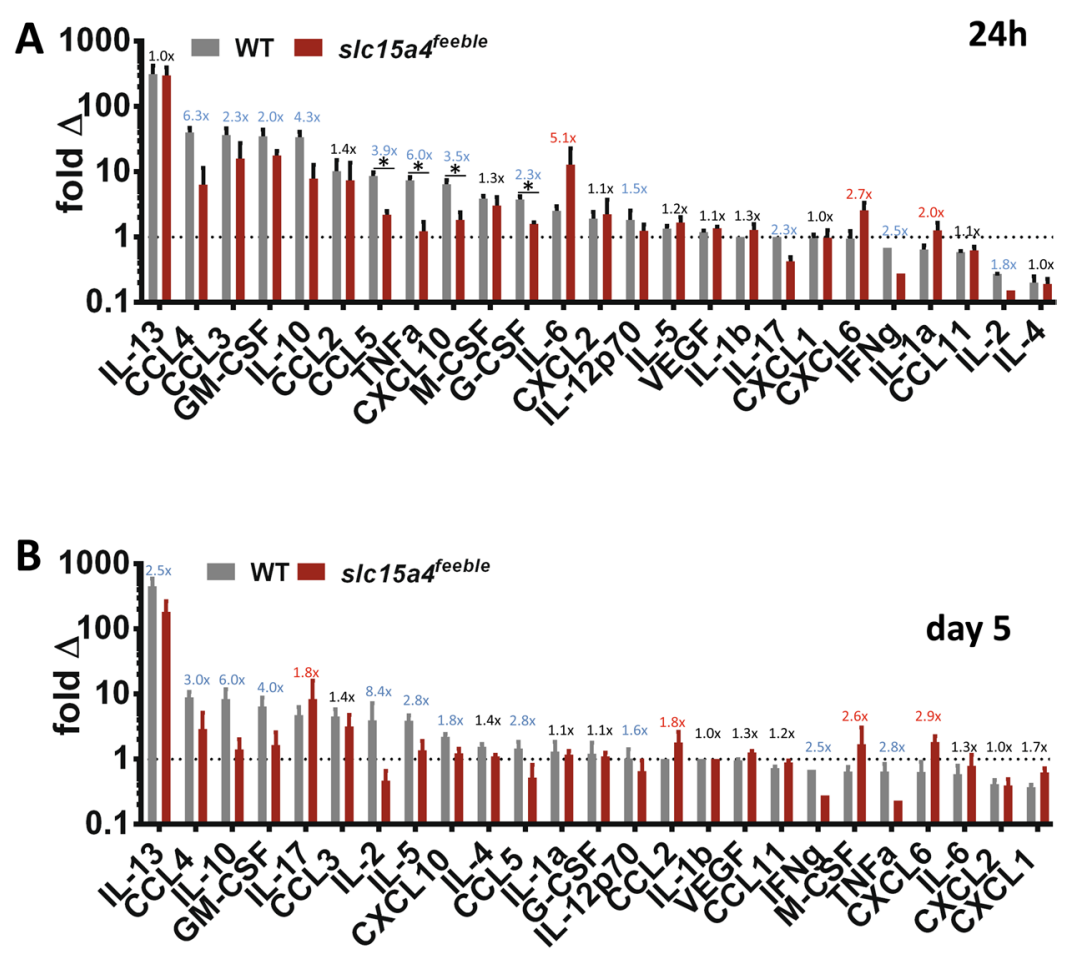

Figure 5. slc15a4 contributes to IMQ driven cytokine storm. Inflammatory cytokine plasma concentrations were measured following IMQ treatment using a 25-cytokine luminex assay. Fold changes (IMQ-treated at the indicated timepoint/time $=0$ ) are shown at 24 hours $(\mathbf{A})$ and 5 days $(\mathbf{B})$. Cytokines were rank-ordered for IMQinduction in WT. The dotted line represents where IMQ treatment does not affect circulating cytokine levels. The ratio of IMQ-induced fold changes are displayed in blue (decreased), red (increased) and black $(<1.5 \mathrm{x}$ changed) comparing mutant to WT. Data was combined from three independent experiments with a total of 5-13 mice per group. ${ }^{*} \mathrm{p}<0.05$ in unpaired T-test after multiple comparison correction.

strategy to specifically disrupt this pathway. These experiments also demonstrate the potential utility of targeting slc15a4 therapeutically. slc15a4feeble mice, lacking pDC sensing, exhibited attenuated local and systemic IMQ effects compared to WT mice.

It is also possible that the effect of $\operatorname{slc} 15 a 4$ deficiency is mediated in part via B cells. However, this is less likely as the reported effects of slc15a4 deficiency in B cells generally occur later than the phenotypes described here in an acute 5 day IMQ model. The only other cell type reported to be affected directly by slc15a4 deficiency is mast cells. However, slc15a4 $4^{-/-}$mast cells degranulate more potently than their WT counterparts ${ }^{38}$ which would not explain our overall observations. 
A
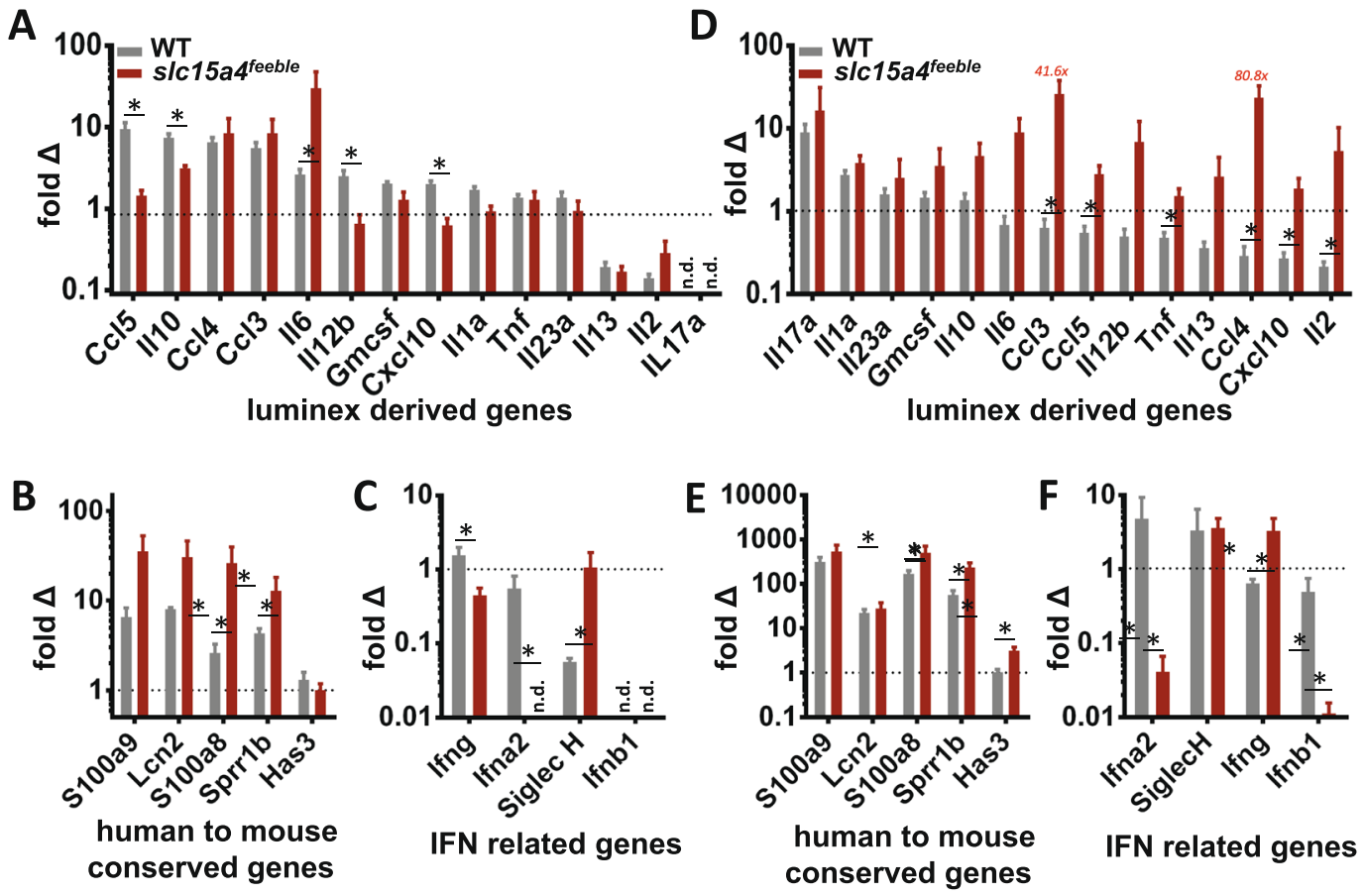

$24 \mathrm{hr}$

day 5

Figure 6. Unexpected more potent induction of trademark psoriasiform genes and inflammatory cytokines over time in skin of slc15a4 deficient mice. RT-qPCR analysis of inflammatory genes elevated in 25plex cytokine analyses in IMQ-treated mice 24 hours (A) and 5 days (D) post treatment with IMQ. IL12b and IL23a were added to address Th1 and Th17 skewing, respectively. Cytokines were rank ordered from greatest to least induction in WT. Expression of trademark psoriasiform genes conserved between human and 5 mouse models is shown rank ordered for human psoriasis at 24 hours (B) and 5 days (E) post-treatment with IMQ. Otherwise, all other datasets are rank ordered for WT mouse induction. IFN-associated genes are shown 24 hours (C) and 5 days $(\mathbf{F})$ post-treatment with IMQ. Fold change $=$ IMQ relative quantity $(\mathrm{RQ}) /$ control RQ, see Materials and Methods for further details. Data is representative of 3 combined experiments with 5-13 mice/group. n.d. $=$ not detected. ${ }^{*} \mathrm{p}<0.05$ in unpaired T-test after multiple comparison correction.

Given the acute effects of IMQ are largely driven via pDC TLR7 sensing ${ }^{28-30}$, we suspect that pDCs mediate the majority of the phenotype in slc15a $4^{\text {feeble }}$. However, the opposite phenotype found in the IL23 model could involve B cells and/or mast cells. These cell types (especially mast cells in the acute setting) may participate in the apparently paradoxical observation of increased inflammasome-related systemic inflammatory cytokines (Fig. 5) and the unexpected increase in cutaneous pro-inflammatory gene expression (Fig. 6). Another explanation for these surprising findings could be inflammatory gene expression compensatory feedback in the absence of IFN-I, the latter of which is likely necessary for acanthosis as IFN-I protein and gene expression was consistently the most decreased pathway in slc15a $4^{\text {feeble }}$ compared to WT mice.

Although slc15a4 is tightly expressed in DC and B cells, we considered the possibility that slc15a4 feeble may have behavioral differences that contributed to our phenotypes ${ }^{19}$. However, no difference in grooming behaviors were observed between $\operatorname{slc} 15 a 4^{f e e b l e}$ and WT mice. Additionally, IMQ was applied until it was fully absorbed via visual inspection to help ensure that any inflammatory differences between WT and mutant mice were not confounded by differential uptake of IMQ or uptake via a different route (i.e. orally).

To understand the basis for the striking absence of weight loss in comparison to the more modest skin changes

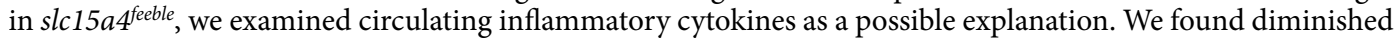
induction of 11/25 and 7/25 IMQ-induced circulating cytokines ( $24 \mathrm{~h}$ and day 5, respectively) that supported this hypothesis (Fig. 5). In addition, the most sustained inflammatory defect in slc15a4 feeble was an absence of IFN-I induction observed at all times at the level of protein and RNA expression when IFN-I is most prominently induced both systemically and locally (Figs 4 and 6). We suspect that the absence of weight loss in slc15a feeble during IMQ treatment is likely due to reduced IFN-I and/or other cytokines in circulation. Strikingly, three cytokines were upregulated at $24 \mathrm{~h}$ and 5 days post IMQ treatment in slc15a feeble versus WT mice. These were all inflammasome-related cytokines ${ }^{32,33,39,40}$. The IMQ psoriasiform model is mediated by the TLR7 vs. inflammasome pathways ${ }^{20}$. slc15a4 is required for TLR7 sensing via pDC consistent with diminished acanthosis, weight loss (Fig. 1), immune cell recruitment (Fig. 3), cytokine storm (Figs 4 and 5) and cutaneous induction of several genes at $24 \mathrm{~h}$ (Fig. 6). However, some inflammation remained in slc15a4 during IMQ topical treatment as measured by acanthosis (Fig. 1) with preserved recruitment of myeloid immune cells (Fig. 3). Our data supports the activation of the inflammasome as a potential etiology for this residual acanthosis whose activation is 
independent of slc15a4 consistent with its reported function. Specifically, the relatively heightened concentration of inflammasome-related cytokines in the plasma (Fig. 5) and inflammasome-related genes in the treated skin (Fig. 6) support a molecular basis for residual IMQ-induced inflammation without pDC-TLR7 sensing. Residual markers of molecular inflammation in $s l c 15 a 4^{\text {feeble }}$ was most striking for the trademark genes whose induction is conserved across human psoriasis and mouse models ${ }^{35}$ at $24 \mathrm{~h}$ and subsequently, most inflammatory cytokines surveyed on day 5 (Fig. 6). Taken together, we reason that pDC-TLR7 sensing can be targeted via slc15a4 and is critical for the inflammatory cascade which results in significant morbidity and weight loss in the IMQ model of psoriasisform dermatitis. In this IMQ-induced model, loss of $s l c 15 a 4$ is most tightly tied to a defect in IFN-I production while an inflammasome signature is preserved.

\section{Materials and Methods}

Mice. All mice used in this study were housed in the Case Western Reserve University Biomedical Research Building Animal Facility. C57BL/6 J mice were bred locally or ordered from Jackson Laboratories. slc15a4feeble/feeble (MGI: 4835997) mutants have been described previously ${ }^{7}$.

Imiquimod challenge model. Briefly, slc15a4 feeble and C57BL/6 J mice were used at 6-8 wks of age and gender did not determine different outcomes. Mouse dorsal skin was treated with IMQ, for 1 day or 5 consecutive days, followed by harvest of tissue and plasma. The dorsal skin of wildtype and feeble mice were shaved prior to IMQ application. $0.05 \mathrm{~g} /$ mouse of IMQ cream (5\%), vehicle only, or no cream was applied to the shaved skin of the mice. Clinical phenotypes (weight loss and acanthosis as described previously ${ }^{19}$ ) were identical between vehicle only and no cream conditions.

IL23 challenge model. Briefly, slc15a4feeble and C57BL/6 J mice were used at 6-8 wks of age and gender did not affect different outcomes. Mice received intralesional injections of $20 \mu \mathrm{l} \mathrm{PBS}$, either alone or containing $500 \mathrm{ng}$ recombinant mouse IL-23 (eBioscience), using a 26-gauge needle every other day for 16 days as previously described $^{41}$. Ear thickness was measured via micrometer before injection on day 0 and thereafter on days without injections.

Immunohistochemistry. Skin was embedded fresh in Tissue Freezing Media (TFM; Triangle Biomedical), flash frozen in liquid nitrogen and immediately stored at $-80^{\circ} \mathrm{C}$. Remaining treated dorsal skin was flash frozen and stored at $-80^{\circ} \mathrm{C}$ for use in RNA experiments. Immunohistochemistry was performed on $8 \mu \mathrm{m}$ sections. Antibodies and isotype controls were detected using either rabbit anti-rat IgG biotinylated or goat anti-rabbit IgG biotinylated, amplified with Avidin/Biotinylated Enzyme Complex (ABC, Vector) and were visualized using the enzyme substrate diaminobenzidine (Vector). Slides were counterstained with hematoxylin and imaged as previously described $^{42}$. Three different observers have quantified each image manually on two occasions.

RNA Isolation and RT-qPCR. Dorsal skin was homogenized in TRIzol using a Mikro-Dismembrator (Sartorius Stedim Biotech, Edgewood, NY) and RNA isolated using RNeasy Mini Kit (Qiagen, Valencia, CA). Transcriptor First Strand cDNA Synthesis Kit (Roche) was performed following the manufacturer's instructions. RT-qPCR was than performed using TaqMan microfluidic array technology from Applied Biosystems on an ABI 7900HT. Relative Quantity (RQ) expression levels were calculated using the comparative CT method $(\Delta \Delta C T)$ and normalized by GAPDH for: Ifn-gamma, Ifn-beta, Siglec H, Gmcsf, Il-2, Il-7, Il-10, Ccl5, Ccl3, Ccl4, Il-13, Il-23, S100a7a, S100a9, S100a8, Cxcl1010, IL-1f6, Tnf-a, Il-12p40, Serpinb3c, IL-1 alpha, Has3, Lcn2, Sprr1b, Pcdh21, Icos, Il-17, Ccl5, Ifna2, and Lor. Absolute values of all measured genes, at baseline and induction, is described in the Supplementary Figures 1,2.

Analysis of circulating cytokines. Plasma collected at $24 \mathrm{~h}$ and 5 days was interrogated with a $25 \mathrm{plex}$ luminex screening assay (R\&D systems) on a Biorad Bioplex Magpix Multiplex Luminex Microplate Reader by the CWRU Bioanalyte core for IL-12p70, VEGF, IL-1beta, CCL2, CXCL2, IL-6, IL-4, IL-2, TNFalpha, CXCL1, IL-17, IL-10, IL-13, IL-5, IFNgamma, CXCL10, CCL5, G-CSF, M-CSF, CCL4, IL-1alpha, CCL3, GM-CSF, CXCL6, and CCL11. IFN-I was measured in plasma by ELISA per manufacturer's instructions (PBL Assay Science). Absolute values of all measured circulating cytokines, at baseline and induction, is described in the Supplementary Figures 3-8.

Statistical Analysis. Unpaired two-sided parametric T-tests were performed between WT and WT + IMQ groups at each time point in addition to comparing slc15a feeble and slc15a feeble + IMQ groups where indicated. When more than 4 groups were compared, statistical significance was determined using the Holm-Sidak method to correct for multiple comparisons with alpha $=5 \%$, as indicated. Each row was analyzed individually, without assuming a consistent $\mathrm{SD}$.

Ethical Approval. Experimental protocols were approved by Case Western Reserve University Institutional Animal Care and Use Committee (IACUC) institutional committee under IACUC protocol \# 2014-0106. Specifically, Less than 5 mice were housed per cage with daily monitoring to ensure optimal animal welfare and to ameliorate suffering. All mouse studies have been conducted according to national and international guidelines and in accordance with institutional regulations governing animal care and useas detailed in IACUC\# 2014-0106.

\section{Data Availability}

The datasets generated during and/or analysed during the current study are available from the corresponding author on reasonable request. 


\section{References}

1. Kurd, S. K. \& Gelfand, J. M. The prevalence of previously diagnosed and undiagnosed psoriasis in US adults: results from NHANES 2003-2004. J Am Acad Dermatol 60, 218-224, https://doi.org/10.1016/j.jaad.2008.09.022 (2009).

2. Gilliet, M. et al. Psoriasis triggered by toll-like receptor 7 agonist imiquimod in the presence of dermal plasmacytoid dendritic cell precursors. Arch Dermatol 140, 1490-1495, https://doi.org/10.1001/archderm.140.12.1490 (2004).

3. Lande, R. et al. Plasmacytoid dendritic cells sense self-DNA coupled with antimicrobial peptide. Nature 449, 564-569, https://doi. org/10.1038/nature06116(2007).

4. Nestle, F. O. et al. Plasmacytoid predendritic cells initiate psoriasis through interferon-alpha production. Journal of Experimental Medicine 202, 135-143, https://doi.org/10.1084/jem.20050500 (2005).

5. Wohn, C. et al. Langerin(neg) conventional dendritic cells produce IL-23 to drive psoriatic plaque formation in mice. Proceedings of the National Academy of Sciences of the United States of America 110, 10723-10728, https://doi.org/10.1073/pnas.1307569110(2013).

6. Swiecki, M. \& Colonna, M. Unraveling the functions of plasmacytoid dendritic cells during viral infections, autoimmunity, and tolerance. Immunological Reviews 234, 142-162, https://doi.org/10.1111/j.0105-2896.2009.00881.x (2010).

7. Blasius, A. L. et al. Slc15a4, AP-3, and Hermansky-Pudlak syndrome proteins are required for Toll-like receptor signaling in plasmacytoid dendritic cells. Proceedings of the National Academy of Sciences of the United States of America 107, 19973-19978, https://doi.org/10.1073/pnas.1014051107 (2010).

8. Sasawatari, S. et al. The Solute Carrier Family 15A4 Regulates TLR9 and NOD1 Functions in the Innate Immune System and Promotes Colitis in Mice. Gastroenterology 140, 1513-1525, https://doi.org/10.1053/j.gastro.2011.01.041 (2011).

9. Yamashita, T. et al. Cloning and functional expression of a brain peptide/histidine transporter. Journal of Biological Chemistry 272, 10205-10211 (1997).

10. Dosenovic, P. et al. Slc15a4 function is required for intact class switch recombination to IgG2c in response to TLR9 stimulation. Immunology and Cell Biology 93, 136-146, https://doi.org/10.1038/icb.2014.82 (2015).

11. Baccala, R. et al. Essential requirement for IRF8 and SLC15A4 implicates plasmacytoid dendritic cells in the pathogenesis of lupus. Proceedings of the National Academy of Sciences of the United States of America 110, 2940-2945, https://doi.org/10.1073/ pnas.1222798110(2013).

12. Han, J. W. et al. Genome-wide association study in a Chinese Han population identifies nine new susceptibility loci for systemic lupus erythematosus. Nat Genet 41, 1234-1237, https://doi.org/10.1038/ng.472 (2009).

13. He, C. F. et al. TNIP1, SLC15A4, ETS1, RasGRP3 and IKZF1 are associated with clinical features of systemic lupus erythematosus in a Chinese Han population. Lupus 19, 1181-1186, https://doi.org/10.1177/0961203310367918 (2010).

14. Wang, C. et al. Genes identified in Asian SLE GWASs are also associated with SLE in Caucasian populations. European Journal of Human Genetics 21, 994-999, https://doi.org/10.1038/ejhg.2012.277 (2013).

15. Lee, H. S. et al. Ethnic specificity of lupus-associated loci identified in a genome-wide association study in Korean women. Annals of the Rheumatic Diseases 73, 1240-1245, https://doi.org/10.1136/annrheumdis-2012-20267 (2014).

16. Zuo, X. B. et al. Variants in TNFSF4, TNFAIP3, TNIP1, BLK, SLC15A4 and UBE2L3 interact to confer risk of systemic lupus erythematosus in Chinese population. Rheumatology International 34, 459-464, https://doi.org/10.1007/s00296-013-2864-3 (2014).

17. Zhang, M. W., Chen, F. R., Zhang, D. M., Zhai, Z. F. \& Hao, F. Association Study Between SLC15A4 Polymorphisms and Haplotypes and Systemic Lupus Erythematosus in a Han Chinese Population. Genetic Testing and Molecular Biomarkers 20, 451-458, https:// doi.org/10.1089/gtmb.2015.0289 (2016).

18. Blasius, A. L., Krebs, P., Sullivan, B. M., Oldstone, M. B. \& Popkin, D. L. Slc15a4, a Gene Required for pDC Sensing of TLR Ligands, Is Required to Control Persistent Viral Infection. Plos Pathogens 8, https://doi.org/10.1371/journal.ppat.1002915 (2012).

19. Hawkes, J. E., Gudjonsson, J. E. \& Ward, N. L. The Snowballing Literature on Imiquimod-Induced Skin Inflammation in Mice: A Critical Appraisal. Journal of Investigative Dermatology 137, 546-549, https://doi.org/10.1016/j.jid.2016.10.024 (2017).

20. Walter, A. et al. Aldara activates TLR7-independent immune defence. Nature Communications 4, https://doi.org/10.1038/ ncomms2566 (2013).

21. Pantelyushin, S. et al. Ror gamma $\mathrm{t}(+)$ innate lymphocytes and gamma delta $\mathrm{T}$ cells initiate psoriasiform plaque formation in mice. Journal of Clinical Investigation 122, 2252-2256, https://doi.org/10.1172/JCI61862 (2012).

22. Grine, L., Dejager, L., Libert, C. \& Vandenbroucke, R. E. Dual Inhibition of TNFR1 and IFNAR1 in Imiquimod-Induced Psoriasiform Skin Inflammation in Mice. Journal of Immunology 194, 5094-5102, https://doi.org/10.4049/jimmunol.1403015 (2015).

23. Gui, J. et al. Therapeutic Elimination of the Type 1 Interferon Receptor for Treating Psoriatic Skin Inflammation. Journal of Investigative Dermatology 136, 1990-2002, https://doi.org/10.1016/j.jid.2016.06.608 (2016).

24. Kader, M. et al. Blocking TLR7-and TLR9-mediated IFN-alpha Production by Plasmacytoid Dendritic Cells Does Not Diminish Immune Activation in Early SIV Infection. Plos Pathogens 9, https://doi.org/10.1371/journal.ppat.1003530 (2013).

25. Kobayashi, T. et al. The Histidine Transporter SLC15A4 Coordinates mTOR-Dependent Inflammatory Responses and Pathogenic Antibody Production. Immunity 41, 375-388, https://doi.org/10.1016/j.immuni.2014.08.011 (2014).

26. Flutter, B. \& Nestle, F. O. TLRs to cytokines: Mechanistic insights from the imiquimod mouse model of psoriasis. European Journal of Immunology 43, 3138-3146, https://doi.org/10.1002/eji.201343801 (2013).

27. Costa, S. et al. Role of MyD88 signaling in the imiquimod-induced mouse model of psoriasis: focus on innate myeloid cells. Journal of Leukocyte Biology 102, 791-803, https://doi.org/10.1002/eji.201343801 (2017).

28. Gibson, S. J. et al. Plasmacytoid dendritic cells produce cytokines and mature in response to the TLR7 agonists, imiquimod and resiquimod. Cell Immunol 218, 74-86, https://doi.org/10.1016/S0008-8749(02)00517-8 (2002).

29. Rajagopal, D. et al. Plasmacytoid dendritic cell-derived type I interferon is crucial for the adjuvant activity of Toll-like receptor 7 agonists. Blood 115, 1949-1957, https://doi.org/10.1182/blood-2009-08-238543 (2010).

30. Hemmi, H. et al. Small anti-viral compounds activate immune cells via the TLR7 MyD88-dependent signaling pathway. Nat Immunol 3, 196-200, https://doi.org/10.1038/ni758 (2002).

31. Seyam, M. S., Freshwater, D. A., O’Donnell, K. \& Mutimer, D. J. Weight loss during pegylated interferon and ribavirin treatment of chronic hepatitis C*. J Viral Hepat 12, 531-535, https://doi.org/10.1111/j.1365-2893.2005.00637.x (2005).

32. Wuyts, A. et al. The CXC chemokine GCP-2/CXCL6 is predominantly induced in mesenchymal cells by interleukin-1beta and is down-regulated by interferon-gamma: comparison with interleukin-8/CXCL8. Lab Invest 83, 23-34 (2003).

33. Netea, M. G., van de Veerdonk, F. L., van der Meer, J. W. M., Dinarello, C. A. \& Joosten, L. A. B. Inflammasome-Independent Regulation of IL-1-Family Cytokines. Annual Review of Immunology Vol 33(33), 49-77, https://doi.org/10.1146/annurevimmunol-032414-112306 (2015).

34. Deng, Y. X., Chang, C. \& Lu, Q. J. The Inflammatory Response in Psoriasis: a Comprehensive Review. Clinical Reviews in Allergy \& Immunology 50, 377-389, https://doi.org/10.1007/s12016-016-8535-x (2016).

35. Swindell, W. R. et al. Genome-Wide Expression Profiling of Five Mouse Models Identifies Similarities and Differences with Human Psoriasis. Plos One 6, https://doi.org/10.1371/journal.pone.0018266 (2011).

36. Johnston, A. et al. IL-1F5, F6, F8, and F9: a novel IL-1 family signaling system that is active in psoriasis and promotes keratinocyte antimicrobial peptide expression. Journal of Investigative Dermatology 130, S43-S43 (2010).

37. Kioon, M. D. A. et al. Plasmacytoid dendritic cells promote systemic sclerosis with a key role for TLR8. Science Translational Medicine 10, https://doi.org/10.1126/scitranslmed.aam8458 (2018). 
38. Kobayashi, T. et al. Lysosome biogenesis regulated by the amino-acid transporter SLC15A4 is critical for functional integrity of mast cells. International Immunology 29, 551-566, https://doi.org/10.1093/intimm/dxx063 (2017).

39. Biondo, C. et al. The Interleukin-1 beta/CXCL1/2/Neutrophil Axis Mediates Host Protection against Group B Streptococcal Infection. Infection and Immunity 82, 4508-4517, https://doi.org/10.1128/IAI.02104-14 (2014).

40. Parry, G. C. N., Martin, T., Felts, K. A. \& Cobb, R. R. IL-1 beta-induced monocyte chemoattractant protein-1 gene expression in endothelial cells is blocked by proteasome inhibitors. Arteriosclerosis Thrombosis and Vascular Biology 18, 934-940, https://doi. org/10.1161/atvb.18.6.934 (1998).

41. Hedrick, M. N. et al. CCR6 is required for IL-23-induced psoriasis-like inflammation in mice. J Clin Invest 119, 2317-2329 (2009).

42. Swindell, W. R. et al. Imiquimod has strain-dependent effects in mice and does not uniquely model human psoriasis. Journal of Investigative Dermatology 137, S111-S111, https://doi.org/10.1186/s13073-017-0415-3 (2017).

\section{Acknowledgements}

This study was made possible by the Skin Diseases Research Center P30AR039750, VA Merit Award IBX002719A, American Skin Association Carson Scholars Fund, the Clinical and Translational Science Collaborative of Cleveland, and UL1TR000439 from the National Center for Advancing Translational Sciences (NCATS) component of the National Institutes of Health and NIH roadmap for Medical Research. These contents are solely the responsibility of the authors and do not necessarily represent the official views of the NIH or other funding sources.

\section{Author Contributions}

A.D.G., A.K.Z., M.H., R.D. and D.L.P. had full access to all of the data in the study and take responsibility for the integrity of the data and the accuracy of the data analysis. Study concept and design: D.L.P. Analysis and interpretation of data: A.D.G., A.K.Z., M.H., R.D., D.L.P. Drafting of the manuscript: A.D.G., A.K.Z., D.L.P. Critical revision of the manuscript for important intellectual content: A.K.Z., R.D., D.L.P. Obtained funding: D.L.P. Administrative, technical, or material support: A.D.G., A.P., J.Y., M.H., R.D. Study supervision: D.L.P.

\section{Additional Information}

Supplementary information accompanies this paper at https://doi.org/10.1038/s41598-018-32668-9.

Competing Interests: The authors declare no competing interests.

Publisher's note: Springer Nature remains neutral with regard to jurisdictional claims in published maps and institutional affiliations.

(c) (i) Open Access This article is licensed under a Creative Commons Attribution 4.0 International License, which permits use, sharing, adaptation, distribution and reproduction in any medium or format, as long as you give appropriate credit to the original author(s) and the source, provide a link to the Creative Commons license, and indicate if changes were made. The images or other third party material in this article are included in the article's Creative Commons license, unless indicated otherwise in a credit line to the material. If material is not included in the article's Creative Commons license and your intended use is not permitted by statutory regulation or exceeds the permitted use, you will need to obtain permission directly from the copyright holder. To view a copy of this license, visit http://creativecommons.org/licenses/by/4.0/.

(C) The Author(s) 2018 